Pensamiento Crítico N. ${ }^{\circ}$ 15, pp. 103-128

\title{
Máximo valor de la empresa y ADRs
}

\author{
Dr. Raimundo Renaun Pacheco Mexzon
}

\section{RESUMEN}

La globalización de la economía y la internacionalización de los mercados de capitales es hoy en día un fenómeno totalmente generalizado en el mundo, por esta razón no hay lugar para aislamiento de los distintos mercados incluyendo el mercado de valores y en el caso del mercado peruano no se escapa a esta realidad. En este nuevo contexto existen en los Estados Unidos de Norte América, los Certificados Americanos de Depósito (American Depositary Recipts) o ADRs.

Los ADRs son instrumentos financieros norteamericanos, cuyo objetivo es permitir que las empresas de países extranjeros puedan en la Bolsa de Valores de New York (New York Stock Exchange: NYSE) colocar sus acciones ingresando de esta manera al mercado de capitales de los Estados Unidos de Norteamérica.

El ingreso al mercado de capital americano, le permite a las empresas de los países extranjeros tener nuevas alternativas de financiamiento empresarial o de diversificación de su accionariado con lo cual amplían las fronteras de sus mercados de capitales. Esta situación es aún más favorable para las empresas de los países emergentes con economías sub desarrolladas por que les permite colocar sus acciones en mercados financieros más evolucionados. 


\section{Pensamiento Crítico N. ${ }^{\circ}$}

La importancia que han tomado estos valores en el mercado bursátil, hace pensar que pueden ser una buena alternativa de financiamiento para las empresas de los países en vías de desarrollo dentro de los cuales se encuentra el Perú. De allí que nuestra inquietud es plantear un modelo sencillo para que la emisión y colocación de ADRs por las empresas peruanas contribuyan a mejorar el valor de la empresa. Este modelo permitió calcular la estructura adecuada de capital y lograr incrementar el valor de la empresa y se concluyó que los ADRs nivel III y nivel IV son una buena alternativa de financiamiento para las empresas peruanas que buscan incrementar su capital y maximizar el valor de mercado de la empresa de acuerdo a una estructura adecuada de capital.

Palabras Claves: ADRs, estructura de capital, máximo valor de la empresa.

\section{ABSTRACT}

The globalization of the economy and the internationalization of capital markets today is quite widespread in the world, that is why there is no place for insulation of different markets, including the stock market and in the case of the Peruvian market is no exception to this reality. In this new context exist in the United States of North America, the American Depositary Recipts or ADRs

The ADRs are U.S. financial instruments, whose goal is to allow companies from foreign countries may in the New York Stock Exchange (NYSE) put their actions by entering in this way to the market of capitals of the United States of America.

Admission to the American capital market, allows companies in foreign countries have new alternatives of business financing or diversification of its shareholders with which expand the borders of their capital markets. This situation is even more favourable for companies in emerging countries with economies sub developed by that allows them to place their actions in financial markets more evolved.

The importance that have taken these values in the stock market, suggests that they may be a good alternative of financing for enterprises in countries developing inside of which is the Peru. From there that our concern is to make a simple model for the issuing and placement of ADRs by Peruvian companies will contribute to improving the value of the company. This model allowed calculate appropriate capital structure and achieve increased the value of the company and it was concluded that the ADRs level III and level IV are a good alternative to financing 


\section{Raimundo Renaun Pacheco Mexzon}

for Peruvian companies seeking to increase their capital and maximizing the market value of the company according to a suitable capital structure.

Keywords: ADRs, Capital Structure, The company's maximum value.

\section{Introducción}

El presente trabajo se desarrolló luego de plantearnos las siguientes preguntas:

¿La emisión de ADRs del nivel III o del nivel IV bajo la norma 144 A que aumenta el capital social, permitiría a las empresas tener una adecuada estructura de capital y maximizar su valor en el mercado?

¿Los ADRs que aumentan el capital social de la empresa pueden generar capital de riesgo al país como inversión directa extranjera?

Para responderlas se desarrolló el marco teórico que incluye la conceptualización de los ADRs y el planteamiento de un modelo que maximiza el valor de la empresa determinando una adecuada estructura de capital.

Luego, se plantea la aplicación del modelo desarrollado en el marco teórico tomando información real de una empresa peruana que cotiza en nuestra Bolsa de Valores de Lima. Esta información que aparece en sus estados financieros auditados, permitió hacer las proyecciones respectivas y llegar a la determinación del valor de la empresa. Con este resultado, y de acuerdo al modelo planteado, se determinó hasta por cual monto se pueden emitir ADRs.

Se concluye que la emisión de ADRs del nivel III y nivel IV son una buena alternativa de financiamiento para las empresas peruanas que buscan incrementar su capital y maximizar el valor de mercado de la empresa de acuerdo a una estructura óptima de capital establecida.

Se recomienda que las empresas peruanas que cumplan con los requisitos exigidos por el mercado norteamericano deban emitir ADRs para lograr financiamiento en condiciones favorables para sus proyectos y mejorar su imagen internacionalmente. 


\section{Pensamiento Crítico N. ${ }^{\circ}$}

Así mismo pueden incrementar su capital trayendo capital fresco de riesgo del exterior al país como inversión directa extranjera.

\section{Marco teórico}

Los ADRs son recibos o certificados americanos de depósito en custodia, nominados en dólares, que representan las acciones emitidas por una empresa no estadounidense, pero que serán negociados en el mercado de valores de dicho país.

Desde 1927 en que el J.P. Morgan introdujo el American depositary receipt, el mercado de los ADRs ha crecido espectacularmente realizándose emisiones por encima de 2200 empresas de más de 80 países del mundo y por montos que superan los 500000 millones de dólares. En el año 2001, se colocaron en este mercado más de 30000 millones y en el primer trimestre del 2002 se negociaron 7500 millones de acciones por un valor de 163000 millones de dólares.

America Telecom y Grupo Electra de México, Sociedad Química y Minera de Chile, Corimon de Venezuela y Quilmes Industrial de Argentina son casos de emisión de ADRs por empresas de países latinoamericanos.

Actualmente están siendo utilizados por algunas empresas peruanas como por ejemplo la compañía de minas Buenaventura, Southern Perú Coper Corporation, Telefónica, Credicorp, Ferreyros y Luz del Sur. que requieren grandes montos de inversión para desarrollar sus proyectos y que generalmente les resulta difícil conseguir estos recursos en el mercado interno. De allí que la importancia de los ADRs para las empresas peruanas radique en que se pueden constituir en una modalidad nueva de financiamiento. Además de participar nuestras empresas en el mercado de capitales norteamericano y mejorar su imagen corporativa, pueden crear valor a la empresa.

Pensar en los ADRs como fuente de financiamiento del crecimiento y desarrollo empresarial del país permite centrarnos en las teorías de la diversificación de las fuentes financieras, en la estructura de capital y en la teoría del valor de la empresa.

Por lo tanto, partiendo de la teoría del valor de la empresa en el mercado, se puede maximizar el valor de la empresa teniendo una estructura adecuada de capital. 


\section{Raimundo Renaun Pacheco Mexzon}

Con esta estructura de capital se determina el patrimonio necesario para maximizar el valor de la empresa y se puede definir cuantas acciones comunes nuevas se pueden emitir y colocar en el mercado americano como ADRs del nivel III o del nivel IV bajo la norma 144 A. De esta manera se estaría trayendo al Perú capital de riesgo como inversión directa extranjera.

La teoría de la diversificación de las fuentes de financiamiento propugna que las empresas deben de buscar utilizar todas las fuentes de financiamiento posibles tanto de corto, mediano y largo plazo de tal manera de tener un costo de capital de promedio ponderado razonable para poder evaluar sus alternativas de inversión en todos sus proyectos.

La obtención de fondos y la determinación de la estructura de capital es una de las funciones a las cuales tiene que hacer frente el Gerente Financiero. Así cuando los egresos de efectivo son mayores que los ingresos, deberá orientar sus esfuerzos hacia la obtención de recursos, dentro o fuera de la empresa. Dentro de la empresa a través del aumento de capital, y fuera de la empresa en el mercado financiero donde se ofertan fondos a diferentes tasas y plazos o a diferentes modalidades de cancelación. Su tarea es lograr la financiación al mínimo costo, determinando al mismo tiempo cuál debe ser la estructura de capital más adecuada para la empresa, es decir cuánto de pasivos y cuánto de capital propio o patrimonio, y de tal manera maximizar el valor de la empresa en el mercado.

El palanqueo financiero considera la capacidad de la empresa para utilizar sus gastos financieros con el objeto de maximizar las ganancias por acción tomando en cuenta los cambios o variaciones en las utilidades. Lógicamente, cuando la empresa no tiene gastos financieros no existirá el palanqueo financiero.

Por consiguiente la teoría exige que si se quiere maximizar las ganancias por acción en una empresa debe existir el financiamiento de terceros y no solo el financiamiento de los activos a través del patrimonio. De allí que algunas veces el palanqueo financiero se calcula mediante la relación pasivo total o deuda total entre el patrimonio neto.

Este ratio llamado también razón de endeudamiento total, indica la relación que existe entre los recursos aportados por terceros o acreedores y los aportados por los dueños o accionistas, determinándose de esta manera la solidez financiera o solvencia financiera de la empresa. 


\section{Pensamiento Crítico N. I5}

Lógicamente, definir una estructura de financiamiento trae consigo un nivel de riesgo financiero que al determinarse como el grado de incertidumbre de no tener la capacidad suficiente para poder cubrir los gastos financieros ante una falta de liquidez, se origina una gran presión de los acreedores cuyas deudas pueden estar impagas.

Al referirnos a la elección de la estructura de capital como un problema por resolver en el mercado para maximizar el valor de la empresa, le estamos planteando al Gerente Financiero encontrar la combinación que le permita a la empresa maximizar su valor en el mercado. Estamos seguros que al maximizarse el valor de la empresa en el mercado también se maximiza la ganancia y riqueza de los accionistas.

Para reafirmar lo planteado en el párrafo anterior, pongamos el ejemplo de una empresa que posee 1000 acciones en circulación a un precio de US\$30 cada una, entonces, el capital propio sería: 1000 X $30=30000$ dólares.

Supóngase que la empresa tiene deudas por US\$15 000, por lo tanto, se puede decir que el valor de mercado de todos los títulos en circulación es:

$30000+15000=45000$ dólares

Si se sustituye 5000 dólares de capital por 5000 de deuda, para pagar un dividendo especial para los accionistas, la nueva estructura sería: 25000 de capital + 20000 de deuda $=45$ 000. En este caso, como no hubo un incremento del valor total, los accionistas han perdido participación por los 5.000. Pero si el valor total hubiera crecido a 50000 como consecuencia de un aporte de capital, la nueva estructura sería: 35000 de capital +15000 de deuda $=50$ 000. En este caso los accionistas se benefician con 5000 de participación por el cambio de la estructura de capital.

De lo expuesto se puede concluir que una política que maximice el valor de mercado de la empresa es también lo mejor para los accionistas.

Por lo tanto, partiendo de la teoría del valor de la empresa en el mercado se puede maximizar el valor de la empresa teniendo una estructura adecuada de capital. 


\section{Raimundo Renaun Pacheco Mexzon}

La representación matemática para calcular el valor de la empresa en el mercado es:

$$
\text { V.E. }=\text { VPN + UT.ACU + PLP + PCP (1) }
$$

Donde:

V.E. $\quad=$ Valor de la empresa en el mercado

VPN $\quad=$ Valor presente neto

UT. ACU. = Utilidades acumuladas

PLP $\quad=$ Pasivos de largo plazo

PCP $=$ Pasivos de corto plazo

Pero se conoce que:

VPN + UT. ACU. $=$ PATRIMONIO

$\mathrm{PLP}+\mathrm{PCP} \quad=$ DEUDA

Reemplazando (2) y (3) en (1), tenemos:

$$
\text { V.E }=\text { PATRIMONIO + DEUDA }
$$

Donde:

V.E. $\quad=$ Valor de la empresa en el mercado

PATRIMONIO $=$ VPN + UT. ACU.

DEUDA $=\mathrm{DLP}+\mathrm{DCP}$

DLP = Deuda de largo plazo

$\mathrm{DCP}=$ Deuda de corto plazo 


\section{Pensamiento Crítico N. ${ }^{\circ}$}

Contando con el marco teórico anteriormente descrito se puede plantear el siguiente MODELO:

$$
\text { Máx. V.E. }=\mathrm{k} * \text { VE (PATRIMONIO) }+\mathrm{w} * \text { V.E. (DEUDA) }
$$

Donde:

El máximo valor de la empresa en el mercado (Max. V. E.) es la variable dependiente y la óptima estructura de capital tiene las variables independientes, PATRIMONIO y DEUDA, que determinan la estructura de capital.

Los coeficientes " $k$ " y "w" son los porcentajes del valor de la empresa que definen el PATRIMONIO y la DEUDA necesarios para determinar la estructura adecuada de capital. Ambos suman "uno" porque representan el 100\%.

Matemáticamente tenemos: $\mathrm{k}+\mathrm{w}=1$ de donde $\mathrm{w}=1-\mathrm{k}$

Luego el máximo valor de mercado de la empresa se da con la siguiente estructura de capital:

$$
\mathrm{k} * \text { V.E. como PATRIMONIO + }(1-\mathrm{k}) * \text { V.E. como DEUDA }
$$

Como se puede apreciar con esta estructura de capital se determina el PATRIMONIO necesario para maximizar el valor de la empresa y se puede definir cuantas acciones comunes nuevas se pueden emitir y colocar en el mercado americano como ADRs del nivel III o del nivel IV bajo la norma 144 A. De esta manera se estaría trayendo al Perú capital de riesgo como inversión directa extranjera.

\section{Objetivo}

El objetivo general del presente trabajo es demostrar que la emisión y colocación de los ADRs del nivel III o del nivel IV bajo la norma 144 A, permitirá a las compañías implementar estos programas y conseguir mejorar el valor de la empresa en el mercado. 


\section{Raimundo Renaun Pacheco Mexzon}

Para lo cual se aplicará un modelo que nos permita calcular la estructura adecuada de capital de una empresa para demostrar que se incrementa valor al emitir los ADRs del nivel III o del nivel IV bajo la norma 144 A mencionados anteriormente, y que por consiguiente se logra inversión directa extranjera.

\section{ADRs}

\subsection{Concepto}

En 1927 un inversionista de Nueva York solicitó a su banquero que le comprara determinadas acciones de una empresa inglesa en la Bolsa de Valores de Londres. Pero le advirtió que no deseaba tener en su contabilidad valores emitidos en libras esterlinas, por lo que le pedía que en representación de las acciones así adquiridas, el banco le emitiera un certificado de depósito expresado en dólares americanos.

Lo que el inversionista registró en su contabilidad fue un certificado de depósito emitido por el banco, y quien en la práctica aparecía como titular de las acciones inglesas era el Banco Depositario. Así fue como funcionó hasta principios de los años 80 con los recibos no auspiciados o no patrocinados.

JP Morgan fue el Banco de Inversión que lanzó el primer ADR para Selfridges Provincial Stores, famosa compradora Británica, el 29 de abril de 1927.

El ADR fue registrado en la New York Curb Exchange precursora de la bolsa de valores de New York.

Con el inusitado crecimiento del mercado en los ochenta intervino la Securities Exchange Comisión (Comisión Nacional de Valores de los Estados Unidos de Norteamérica: SEC) y se creó la regla conocida como 129. Esta regla, sobre excepción de información, permitió la emisión de ADRs como certificados representativos de acciones.

La empresa emisora de las acciones, aunque no esté registrada en la Bolsa de Valores dentro de los Estados Unidos, está obligada a suministrar a la SEC toda la información y documentación contable que obligatoriamente presenta a las autoridades de su país. Debe también presentar el contrato firmado con el banco norteamericano que actuará como depositario. 


\section{Pensamiento Crítico N. ${ }^{\circ}$}

Este fue el origen de la emisión de ADRs conocida como emisión auspiciada nivel I.

Los ADRs (American Depositary Receipts) son recibos o certificados negociables emitidos por un banco norteamericano que representa la posesión de títulos (acciones comunes) que han sido originalmente emitidos por una empresa extranjera y que se negocian en el mercado de capitales de los Estados Unidos de Norteamérica.

Los ADRs que son emitidos por los bancos norteamericanos están respaldados por las acciones emitidas por una empresa extranjera quien a través de su banco custodio en su país de origen facilitan la transferencia física de las acciones al banco depositario de los Estados Unidos.

\subsection{Clases o programas de ADRs}

Si quisiéramos conocer algo más de los ADRs, podemos decir que existen dos clases o tipos de programas:

a) Los programas auspiciados.

b) Los programas no auspiciados.

Los programas auspiciados involucran a un solo banco depositario quien emite los ADRs de conformidad con el contrato firmado con la empresa extranjera emisora de las acciones que sirven de respaldo a los ADRs. Hoy en día las empresas bancarias y demás compañías que están autorizadas a emitir ADRs, prefieren trabajar con programas auspiciados, lo que implica tener un solo banco depositario con el cual se mantiene un estrecho control sobre los valores transados en el mercado de capitales norteamericano.

Los programas no auspiciados se caracterizan por que la emisión de los ADRs la realizan uno o más bancos depositarios. En este caso no existe un contrato formal entre la empresa extranjera emisora de las acciones y los bancos depositarios. No hay una exclusividad en el manejo por lo cual la empresa emisora pierde en algunos casos el control sobre las colocaciones de sus acciones. 


\section{Raimundo Renaun Pacheco Mexzon}

Este tipo de programas origina una pérdida de control debido a que se negocian en el mercado extrabursatil llamado Over the Counter (OTC) donde se realizan las transacciones entre vendedores y compradores utilizando para ello mecanismos fuera de bolsa.

Por lo general los precios de los ADRs de estos programas no auspiciados son manejados y publicados en la National Automated Quotation System (NASDAQ) cuya distribución están restringidas a Brokers o intermediarios mas no al público en general.

Estos programas no son elegibles para manejarse en bolsa y la emisión de los ADRs están respaldados por emisiones de acciones que ya se realizaron anteriormente por lo que no apoyan a la empresa en lograr un aumento de capital.

La SEC ha establecido una serie de requisitos para las empresas que tienen programas no auspiciados dificultando su aplicación. Esto ha ocasionado una disminución en el uso de estos valores y están con clara tendencia a su desaparición.

\subsection{Tipos de programas auspiciados}

Existen cuatro tipos de programas auspiciados. Estos se clasifican por niveles, dos de ellos están diseñados para que la empresa pueda colocar en el mercado de capitales sus acciones ya emitidas con anterioridad y los otros dos para que se puedan colocar emisiones primarias tendientes a incrementar el capital.

\section{Programa Nivel I}

Este programa se realiza sobre la base de acciones que ya se encuentran en circulación en el país de origen de la empresa emisora.

Su negociación secundaria se realiza en el mercado extrabursatil OTC a través de las páginas rosadas donde se muestran los precios de estos valores y toda la información referente a la empresa y los intermediarios que trabajan con estos valores. 


\section{Pensamiento Crítico N. 15}

El principal propósito de este programa es que las empresas extranjeras se hagan conocidas en el mercado norteamericano.

Las ventajas de este programa se pueden resumir en:

1. La empresa emisora escoge el banco depositario manteniendo el control sobre todo el programa.

2. Hay comunicación fluida entre la empresa y los tenedores de los ADRs

3. Los costos son asumidos por los inversionistas

4. Permite acceder con mayor facilidad a los otros programas en caso la empresa así lo decida.

Las desventajas se resumen en:

1. Las acciones no son inscritas en las Bolsa de Valores Norteamericanas.

2. El programa se desarrolla en un mercado poco líquido por que se efectúa a través de la Compañía Central de Depósito (Depositary Trust Company: DTC).

3. No se permite el incremento de capital con este programa.

Para implementar este programa se deben cumplir con los siguientes requisitos:

1. Obtener de la SEC la aprobación de la solicitud de excepción que permite a la empresa emisora presentar solo la información a la cual está obligada en su país de origen.

2. Suscribir un acuerdo de depósito entre la empresa emisora y el banco depositario

3. Llenar el formato F-6 dado por la SEC para que se registren los ADRs

4. Contar con un broker en el mercado norteamericano 


\section{Raimundo Renaun Pacheco Mexzon}

\section{Programa Nivel II}

Al igual que el programa de nivel I, se realiza sobre las acciones que se encuentran en circulación en el país de origen. Sin embargo, se transan las operaciones en la Bolsa de Valores de New York (New York Stock Exchange: NYSE) o en el Sistema de Negociación Automática de Intermediarios de Valores (NASDAQ), razón por la cual se permite que mayor número de inversionistas tengan acceso a estos valores.

En razón de su registro en Bolsa, la empresa emisora necesita presentar información más detallada que la exigida en el nivel I . Así mismo, debe conciliar sus estados financieros con los principios de contabilidad generalmente aceptados en los Estados Unidos. Este programa cuesta más en su implementación pero permite lograr un mercado secundario más amplio.

Dentro de sus ventajas se pueden mencionar las siguientes:

1. Los valores se negocian en Bolsa que son muy buenos mercados y de mayor liquidez.

2. Proporcionan mejor flujo de información financiera del mercado y del emisor.

3. Mayor prestigio para la empresa emisora por negociar valores de mayor liquidez.

Dentro de las desventajas se pueden menciona las siguientes:

1. Requiere información mas detallada.

2. Hay que conciliar los estados financieros de la empresa con los principios generalmente aceptados de contabilidad que se utilizan en los Estados Unidos de Norteamérica.

3. La elaboración del programa es más costoso y demora más tiempo su implementación.

4. No se permite utilizar estos ADRs para incremento de capital. 


\section{Pensamiento Crítico N. I5}

Los requisitos que hay que tener en cuenta son:

1. Presentar para la aprobación de la SEC, el formato 20-F que incluye los datos detallados de la emisión y los estados financieros preparados de acuerdo a los principios generalmente aceptados.

2. Suscribir un acuerdo de depósito entre el emisor y el banco depositario.

3. Gestionar la inscripción en Bolsa a través del formato F-6.

4. Completar los requerimientos para la negociación y liquidación de los ADRs.

5. Preparar los certificados y solicitar el código de estos valores.

\section{Programa Nivel III}

Está diseñado para que las empresas emisoras obtengan capital del mercado norteamericano a través de una oferta pública de ADRs. Tanto los ADRs como las acciones que los respaldan deben estar inscritos en la Bolsa. Normalmente se necesita de un banco de inversión para que actúe como subscriptor y colocador de los valores. El banco de inversión puede adquirir él mismo toda la emisión al participar con una operación llamada underwriting.

Las ventajas que brinda este programa se resumen en:

1. Permite a la empresa emisora conseguir recursos considerados como aportes de capital, al negociar en el mercado de capitales de los Estados Unidos la emisión primaria de sus acciones que respaldan la emisión de los ADRs.

2. Brinda mayor confianza al inversionista norteamericano debido a que le son aplicadas todas las normas establecidas en los Estados Unidos de Norteamérica, según acta de 1933 y 1934 de la ley de la Bolsa de Valores.

3. Tienen muy buena perspectivas de colocación debido a que se cotizan en los mercados más grandes y líquidos del mundo. 


\section{Raimundo Renaun Pacheco Mexzon}

Las desventajas se pueden resumir en:

1. Se exige mayor detalle de la información a presentar a la SEC que lo que se establece para los programas de nivel I y nivel II.

2. Demanda mayor trabajo y costos en la preparación y presentación de los reportes financieros utilizando el formato $20-\mathrm{F}$.

Los requisitos a tener en cuenta son:

1. Preparar y presentar para su aprobación por la SEC, toda la información incluida en el formato 20-F.

2. Suscribir un acuerdo de depósito entre el banco depositario de las acciones que respaldan la emisión de los ADRs y la empresa extranjera que emite las acciones.

3. Presentar la solicitud de registro en Bolsa y conseguir su aprobación.

4. Presentar toda la información solicitada en el formato F-6.

\section{Programa Nivel IV}

Estos programas de ADRs están destinados a respaldar la emisión primaria de acciones con miras a lograr un incremento de capital.

Al igual que los programas de nivel III, son utilizados cuando la empresa emisora requiere de un aumento de capital, sin embargo su colocación están bajo la norma $144 \mathrm{~A}$.

La norma 144 A establece que la colocación de estos valores debe hacerse en exclusividad a los inversionistas institucionales calificados (Qualified Institutional Buyer: QUIBs), como son: empresas administradoras de fondos mutuos, de fondos de pensiones, entidades de ahorro y crédito, compañías de seguros, empresas de inversión, entre otras. 


\section{Pensamiento Crítico N. ${ }^{\circ}$}

Dentro de sus ventajas se pueden mencionar las siguientes:

1. La información financiera a presentar no tiene el mismo nivel de rigor que para los programas de nivel III.

2. Su implementación son de menor costo que de los programas de nivel II y III.

3. Existen alrededor de 5000 QUIBs en el mercado que representan potenciales demandantes de estos valores.

4. Dentro de sus desventajas destaca que la negociación de estos valores debe ser directamente a los QUIEBs lo cual es una limitación.

Dentro de los requisitos podemos mencionar los siguientes:

1. Presentar la solicitud de excepción bajo la regla 12g, 3-2 (b) de la norma $144 \mathrm{~A}$.

2. Tener suscrito el acuerdo de depósito con el banco depositario.

\section{Un caso práctico}

En esta parte del trabajo se escogerá una empresa que actualmente cotiza en la Bolsa de Valores de Lima y que cumpla con las exigencias del caso para emitir ADRs y se le aplicará el modelo planteado en el marco teórico.

De esta manera se determinará su estructura adecuada de capital que permita maximizar su valor en el mercado, definiéndose cuántas acciones comunes nuevas tiene que emitir para que sirvan de respaldo a la emisión de los ADRs.

La colocación en la Bolsa de New York de estos ADRs, permitirá traer capital de riesgo como inversión directa extranjera al país. 


\section{RESUMEN HISTÓRICO DEL BALANCE GENERAL}

en miles de soles

\begin{tabular}{|c|c|c|c|c|}
\hline & AÑO 4 & AÑO 3 & AÑO 2 & AÑO 1 \\
\hline ACTIVO CORRIENTE & 475,171 & 473,222 & 739,462 & 607,125 \\
\hline Caja/Bancos & 91,968 & 28,102 & 232,676 & 58,132 \\
\hline Cuentas por cobrar comercio & 69,542 & 100,420 & 126,657 & 125,670 \\
\hline Otras cuentas por cobrar & 94,276 & 121,327 & 140,169 & 158,699 \\
\hline Existencias & 201,385 & 223,373 & 239,960 & 264,624 \\
\hline ACTIVO NO CORRIENTE & $2,037,979$ & $1,917,982$ & $1,618,248$ & $1,834,445$ \\
\hline Activo fijo neto & 802,900 & 801,759 & 861,694 & 902,734 \\
\hline Otras inversiones & 864,976 & 878,378 & 617,212 & 781,043 \\
\hline Intangibles netos & 370,103 & 237,845 & 139,342 & 150,668 \\
\hline TOTAL ACTIVOS & $2,495,150$ & 2,391204 & $2,357,716$ & $2,441,570$ \\
\hline PASIVO CORRIENTE & 301,160 & 303,071 & 422,896 & 399,989 \\
\hline Préstamos bancarios & 17,273 & 83,704 & 25,076 & 107,454 \\
\hline Cuentas por pagar comercio & 23,445 & 28,046 & 29,166 & 41,982 \\
\hline Otras cuentas por pagar & 156,754 & 120,210 & 129,088 & 155,383 \\
\hline Parte corriente DLP & 103,688 & 71,111 & 239,566 & 95,165 \\
\hline PASIVO NO CORRIENTE & 534,896 & 602,882 & 229,354 & 322,627 \\
\hline Deuda a L.P. & 512,405 & 576,641 & 199,546 & 315,772 \\
\hline Otros pasivos & 22,491 & 26,246 & 29,808 & 6,855 \\
\hline TOTAL PASIVOS & 836,056 & 905,958 & 652,250 & 722,616 \\
\hline PATRIMONIO & $1,659,094$ & $1,485,246$ & $1,705,460$ & $1,718,954$ \\
\hline Capital social & $1,003,482$ & $1,003,482$ & $1,003,482$ & 967,227 \\
\hline Acciones de inversión & 491,174 & 491,174 & 491,174 & 473,429 \\
\hline Reservas y otros & 140,316 & $(37,005)$ & 185,286 & 203,542 \\
\hline Resultados acumulados & 24,122 & 27,595 & 25,518 & 74,756 \\
\hline $\begin{array}{l}\text { TOTAL PASIVOS Y } \\
\text { PATRIMONIO }\end{array}$ & $2,495,150$ & $2,391,204$ & $2,357,710$ & $2,441,570$ \\
\hline
\end{tabular}


Con estos datos históricos podemos presentar el comportamiento que han tenido los coeficientes " $k$ " $y$ " $w$ " en el periodo del año 1 al año 4 conforme se indica a continuación:

\begin{tabular}{|c|c|c|c|c|}
\hline COEFICIENTE & AÑO 4 & AÑ̃ 3 & AÑO 2 & AÑ̃ 1 \\
\hline "k" & 0,66 & 0,62 & 0,72 & 0,70 \\
\hline "w" & 0,34 & 0,38 & 0,28 & 0,30 \\
\hline "k"+ "w" & 1,00 & 1,00 & 1,00 & 1,00 \\
\hline
\end{tabular}

Del comportamiento de los coeficientes " $k$ " $y$ "w" podemos inferir que, de acuerdo a los resultados obtenidos por la empresa, la estructura de capital ha sufrido modificaciones muy importantes pasando de una relación de 70\% de capital y 30\% de deuda en el año 1 , a una estructura de capital de $66 \%$ de patrimonio y $34 \%$ de deuda en el año 4.

A continuación se presenta el resumen del estado de ganancias y pérdidas para el mismo periodo.

RESUMEN HISTÓRICO DEL ESTADO DE GANANCIAS Y PÉRDIDAS En miles de soles

\begin{tabular}{|c|c|c|c|c|}
\hline RUBROS & AÑO 4 & AÑO 3 & AÑO 2 & AÑO 1 \\
\hline Ventas netas & 865,787 & 870,716 & 892,863 & 982,512 \\
\hline Otros ingresos & 38,002 & 27,381 & 37,086 & 45,734 \\
\hline TOTAL INGRESOS & $\mathbf{9 0 3 , 7 8 9}$ & $\mathbf{8 9 8 , 0 9 7}$ & $\mathbf{9 2 9 , 9 4 9}$ & $\mathbf{1 , 0 2 8 , 2 4 6}$ \\
\hline Costo de ventas & 332,528 & 350,592 & 374,410 & 401,080 \\
\hline Utilidad bruta & $\mathbf{5 7 1 , 2 6 1}$ & $\mathbf{5 4 7 , 5 0 5}$ & $\mathbf{5 5 5 , 5 3 0}$ & $\mathbf{6 2 7 , 1 6 6}$ \\
\hline Gastos operacionales & 312,570 & 325,213 & 365,605 & 381,089 \\
\hline Utilidad operativa & $\mathbf{2 5 8 , 6 9 1}$ & $\mathbf{2 2 2 , 2 9 2}$ & $\mathbf{1 8 9 , 9 3 4}$ & $\mathbf{2 4 6 , 0 7 7}$ \\
\hline Otros ingresos y egresos & 17,525 & 36,548 & 59,852 & 59,787 \\
\hline $\begin{array}{c}\text { Utilidad antes de } \\
\text { participación }\end{array}$ & $\mathbf{1 9 1 , 1 6 6}$ & $\mathbf{1 8 5 , 7 4 4}$ & $\mathbf{2 4 9 , 7 8 6}$ & $\mathbf{1 8 6 , 2 9 0}$ \\
\hline Participaciones & 22,581 & 20,468 & 21,251 & 19,091 \\
\hline $\begin{array}{c}\text { Utilidad antes de } \\
\text { impuestos }\end{array}$ & $\mathbf{1 6 8 , 5 8 5}$ & $\mathbf{1 6 5 , 2 7 6}$ & $\mathbf{2 2 8 , 5 3 5}$ & $\mathbf{1 6 7 , 1 9 9}$ \\
\hline Impuesto a la renta & 52,010 & 54,987 & 44,794 & 51,233 \\
\hline Utilidad neta & $\mathbf{1 1 6 , 5 7 5}$ & $\mathbf{1 1 0 , 2 8 9}$ & $\mathbf{1 8 3 , 7 4 1}$ & $\mathbf{1 1 5 , 9 6 6}$ \\
\hline
\end{tabular}




\section{Raimundo Renaun Pacheco Mexzon}

De acuerdo a los datos históricos, se aprecia que los ingresos por ventas han tenido un comportamiento cíclico llegando en el año 4 a 865 millones 787 mil soles. La utilidad operativa llegó a 258 millones 691mil soles y la utilidad neta a 116 millones 575 mil soles.

Tomando como base la data histórica, se calculará el valor de la empresa, en dólares, considerando un escenario futuro de cinco años y las siguientes políticas:

Mantener el crecimiento de la utilidad antes de intereses y de impuestos, para lo cual, deben crecer las ventas de tal manera que de llegar al nivel que se alcanzó en el año 1 (aproximadamente 273 millones de dólares). El crecimiento de las ventas tendrá el siguiente comportamiento:

\section{PROYECCIONES DE LAS VENTAS (EN MILES DE DÓLARES)}

\begin{tabular}{|c|c|c|c|c|c|c|}
\hline AÑOS & $\mathbf{0}$ & $\mathbf{1}$ & $\mathbf{2}$ & $\mathbf{3}$ & $\mathbf{4}$ & $\mathbf{5}$ \\
\hline Ventas & 240,441 & 252,463 & 257,512 & 262,662 & 267,916 & 273,274 \\
\hline Crecimiento & & $5 \%$ & $2 \%$ & $2 \%$ & $2 \%$ & $2 \%$ \\
\hline
\end{tabular}

El costo de ventas que ha tenido un comportamiento entre el $41 \%$ y el $38 \%$ de las ventas, debe llegar como máximo a un $40 \%$. Por lo tanto el costo de ventas tendrá el siguiente comportamiento:

\section{PROYECCIONES DEL COSTO DE VENTAS EN MILES DE DÓLARES}

\begin{tabular}{|c|c|c|c|c|c|}
\hline AÑOS & $\mathbf{1}$ & $\mathbf{2}$ & $\mathbf{3}$ & $\mathbf{4}$ & $\mathbf{5}$ \\
\hline Costo de ventas & 100,985 & 103,005 & 105,065 & 107,166 & 109,310 \\
\hline $\begin{array}{c}\text { Porcentaje de } \\
\text { ventas }\end{array}$ & $40 \%$ & $40 \%$ & $40 \%$ & $40 \%$ & $40 \%$ \\
\hline
\end{tabular}

Los gastos operativos que han tenido un comportamiento entre $36 \%$ y $41 \%$ de las ventas, deben llegar como máximo a un $38 \%$ del costo de venta, por lo tanto tendrán el siguiente comportamiento: 


\section{PROYECCIONES DE LOS GASTOS OPERATIVOS EN MILES DE DÓLARES}

\begin{tabular}{|c|c|c|c|c|c|}
\hline AÑOS & $\mathbf{1}$ & $\mathbf{2}$ & $\mathbf{3}$ & $\mathbf{4}$ & $\mathbf{5}$ \\
\hline Gastos operativos & 38,374 & 39,142 & 39,925 & 40,723 & 41,538 \\
\hline $\begin{array}{c}\text { Porcentaje del } \\
\text { costo de ventas }\end{array}$ & $38 \%$ & $38 \%$ & $38 \%$ & $38 \%$ & $38 \%$ \\
\hline
\end{tabular}

El capital de trabajo debe de crecer acorde con las ventas es decir $5 \%$ el primer año y $2 \%$ del segundo al quinto año. Por lo tanto, el crecimiento del capital de trabajo será:

\section{CRECIMIENTO DEL CAPITAL DE TRABAJO EN MILES DE DÓLARES}

\begin{tabular}{|c|c|c|c|c|c|}
\hline AÑOS & $\mathbf{1}$ & $\mathbf{2}$ & $\mathbf{3}$ & $\mathbf{4}$ & $\mathbf{5}$ \\
\hline $\begin{array}{c}\text { Incremento del } \\
\text { K. Tra. }\end{array}$ & 2,167 & 2,210 & 2,254 & 2,299 & 2,345 \\
\hline
\end{tabular}

Históricamente, la empresa invirtió en la modernización de sus instalaciones 30 millones de soles, equivalente a 8 millones 333 mil dólares. Este tipo de inversión, que es necesaria para mantener sin problemas el proceso productivo, se estima que la empresa la ejecutará en el tercer año, incrementando de esta manera el activo fijo. La depreciación de este tipo de activos se realizará en 5 años a razón de $20 \%$ anual.

Con esta información el flujo de caja libre generado por la empresa en los próximos cinco años sería:

\section{FLUJO DE CAJA LIBRE PROYECTADO \\ EN MILES DE DÓLARES}

\begin{tabular}{|c|c|c|c|c|c|}
\hline AÑOS & $\mathbf{1}$ & $\mathbf{2}$ & $\mathbf{3}$ & $\mathbf{4}$ & $\mathbf{5}$ \\
\hline Ventas & 252,463 & 257,512 & 262,662 & 267,916 & 273,274 \\
\hline Costo de Ventas & 100,985 & 103,005 & 105,065 & 107,166 & 109,310 \\
\hline Gastos Operativos & 38,374 & 39,142 & 39,925 & 40,723 & 41,538 \\
\hline $\begin{array}{c}\text { Utilidad Antes } \\
\text { de Intereses e } \\
\text { Impuestos }\end{array}$ & 113,103 & 115,365 & 117,673 & 120,026 & 122,427 \\
\hline
\end{tabular}




\section{Raimundo Renaun Pacheco Mexzon}

\begin{tabular}{|c|c|c|c|c|c|}
\hline $\begin{array}{c}\text { Utilidad antes } \\
\text { de intereses } \\
\text { después de } \\
\text { impuestos } \\
\text { (Tasa 30\%) }\end{array}$ & $\mathbf{7 9 , 1 7 2}$ & $\mathbf{8 0 , 7 5 6}$ & $\mathbf{8 2 , 3 7 1}$ & $\mathbf{8 4 , 0 1 8}$ & $\mathbf{8 5 , 6 9 9}$ \\
\hline (+) Depreciación & 9,888 & 9,888 & 9,888 & 11,554 & 11,556 \\
\hline $\begin{array}{c}\text { (-) Desembolso } \\
\text { de capital por } \\
\text { inversión }\end{array}$ & 9,888 & 9,888 & 18,221 & 11,554 & 11,556 \\
\hline $\begin{array}{c}\text { (-) Incremento K. } \\
\text { trabajo }\end{array}$ & 2,167 & 2,2102 & 2,254 & 2,299 & 2,345 \\
\hline $\begin{array}{c}\text { Flujo de } \\
\text { caja libre }\end{array}$ & $\mathbf{7 7 , 0 0 6}$ & $\mathbf{7 8 , 5 4 6}$ & $\mathbf{7 1 , 7 8 3}$ & $\mathbf{8 1 , 7 1 9}$ & $\mathbf{8 3 , 3 5 3}$ \\
\hline
\end{tabular}

Para traer a valor presente el flujo de caja libre debemos de calcular previamente el costo promedio ponderado de capital (WACC) utilizando la siguiente fórmula:

$$
\mathrm{WACC}=[\mathrm{P} /(\mathrm{P}+\mathrm{D})] *\left(\mathrm{~T} \operatorname{lr}+\mathrm{B}^{*} \mathrm{PRM}\right)+[\mathrm{D} /(\mathrm{P}+\mathrm{D})] * \mathrm{kd}(1-\mathrm{T})
$$

\section{CÁlCULO DEL COSTO DEL CAPITAL PROPIO}

Para calcular el costo del capital propio se suele utilizar las condiciones presentadas en el mercado considerándose los riesgos respectivos. Es a partir del modelo CAPM (Capital Assets Pricing Model) que se puede calcular una tasa de rendimiento esperado (Tresp) para el accionista, que estaría definiendo su costo de oportunidad. El modelo que se resume en la siguiente fórmula nos permitió calcular el costo del capital propio.

$$
\text { Tresp }=\mathrm{TLr}+\mathrm{B}(\mathrm{RPM}-\mathrm{TLr})
$$

TLr es la tasa libre de riesgo y se asumió que su valor en el mercado es de 5,53\% tomando en consideración el rendimiento que pagan los "Treasury Bill" en el mercado americano. 


\section{Pensamiento Crítico N. ${ }^{\circ}$}

RPM - TLr es la prima del mercado (PRM) y para el caso de empresas peruanas está relacionada con la diferencia entre lo que pagan los Bonos Brady y la tasa del Treasury Bill. En este caso se asumió que es de 5,7\% tomando en cuenta que los Bonos Brady pagan 11,23\% y el Treasury Bill 5,53\%. Además, hay que tener en cuenta que en el Perú para cualquier actividad se podría utilizar este valor ya que en el mercado americano se considera como prima de riesgo y ha sido calculado como el promedio geométrico del índice S \& P 500.

"B" conocido como beta, es el factor que determina las condiciones de riesgo con las probabilidades de rendimientos esperados en el mercado a través de la relación de la covarianza y la varianza. En el mercado americano se estima para las empresas del sector de extracción de recursos naturales y sin apalancamiento un beta promedio de 1.7, sin embargo, para empresas líderes, el beta oscila entre -1 y 1.2 , dependiendo del riesgo. Como nuestra empresa es una empresa líder en el Perú, el beta considerado es de 1.06 que resulta de ajustar 1.7 a un nivel de riesgo manejable del 62.35\%. ( $1.7^{*}$ $0.6235=1.06$ )

En consecuencia el costo del capital propio para nuestra empresa se estimó en:

$$
\begin{aligned}
& \text { Tresp. }=\text { TLr }+ \text { B }(\text { RPM }- \text { TLr }) \\
& \text { Tresp. }=5.53+1.06 *(11.23-5.53)
\end{aligned}
$$

$$
\text { Tresp. }=11.57 \%
$$

\section{CÁlCULO DEL COSTO DE LA DEUDA}

Para calcular el costo de la deuda se han tomado los datos históricos de los estados financieros. Se determinó una tasa en soles de $9.45 \%$ anual. Esta tasa ajustada a la tasa equivalente en dólares, nos dio una tasa de $3.15 \%$. En consecuencia la tasa para la deuda se estimó en $3.15 \%$ anual. 


\section{Raimundo Renaun Pacheco Mexzon}

\begin{tabular}{|l|c|}
\hline \multicolumn{1}{|c|}{ RUBROS } & MILES DE SOLES \\
\hline Gastos financieros & 69,191 \\
\hline Total deuda & 731,456 \\
\hline Sobregiros y préstamos bancarios & 83,704 \\
\hline Vencimiento corriente deuda largo plazo & 71,111 \\
\hline Deuda a largo plazo & 576,641 \\
\hline Gastos financieros entre deuda & $9.45 \%$ \\
\hline Costo promedio deuda en US\$ & $6.10 \%$ \\
\hline Tasa equivalente en dólares & $(1+9.45 \%) /(1.061)=3.15 \%$ \\
\hline
\end{tabular}

De acuerdo a la información obtenida los valores para calcular el WACC son:

$$
\begin{array}{ll}
\mathrm{P} /(\mathrm{P}+\mathrm{D}) & =66 \% \\
\mathrm{D} /(\mathrm{P}+\mathrm{D}) & =34 \% \\
\mathrm{Tlr} & =5.53 \% \\
\mathrm{~B} & =1.06 \\
\mathrm{PRM} & =5.70 \% \\
\mathrm{Kd} & =3.15 \% \\
\mathrm{~T} & =30 \%
\end{array}
$$

Al reemplazar los datos en la fórmula se obtiene un WACC de $8.39 \%$. De esta manera el valor actual del flujo de caja libre se calcula de la siguiente manera:

\section{VALOR ACTUAL DEL FLUJO LIBRE}

\begin{tabular}{|c|c|c|c|}
\hline AÑOS & FLUJO & FACTOR 8.39 \% & V.A. \\
\hline 1 & 77,006 & 0.92262 & 71,047 \\
\hline 2 & 78,546 & 0.85122 & 66,860 \\
\hline 3 & 71,783 & 0.78535 & 56,375 \\
\hline 4 & 81,719 & 0.72458 & 59,212 \\
\hline 5 & 83,353 & 0.66851 & 55,723 \\
\hline TOTAL VA & & & $\mathbf{3 0 9 , 2 1 7}$ \\
\hline
\end{tabular}




\section{Pensamiento Crítico N. 15}

Como la empresa es un negocio en marcha se estima que a partir del sexto año habrá un flujo continuo con un crecimiento de las ventas en $1 \%$ por lo tanto para el año 6 el flujo libre será de 83,353*1.01 = 84,187. Así mismo, el retorno sobre la nueva inversión será de $1 \%$ en forma continua:

Con esta información se calcula el valor continuo que será igual a:

$\mathrm{VC}=\mathrm{FC}$ Libre año $6 /$ (WACC - Retorno nueva inversión)

$\mathrm{VC}=84,187 /(8.39 \%-1 \%)$

$\mathrm{VC}=1,139$ millones 628 mil dólares

Como el costo de capital promedio ponderado (WACC) es $8.39 \%$ el Valor presente o valor actual de este flujo continuo sería:

$\mathrm{VAVC}=1139628000 /(1+0,0839) \wedge 6$

$$
\text { VAVC }=702 \text { millones, } 902 \text { mil dólares }
$$

Luego el valor de la empresa será:

V.E. $=$ VA + VAVC

V.E. $=309217+702902$

$$
\mathrm{VE}=1012 \text { millones } 118 \text { mil dólares }
$$

Con este resultado y de acuerdo al modelo planteado se pueden emitir ADRs hasta por 210 millones de dólares, respetando la estructura financiera determinada que es de $66 \%$ de capital y $34 \%$ de deuda. El cálculo se presenta en el siguiente cuadro. 


\section{Raimundo Renaun Pacheco Mexzon}

\begin{tabular}{|l|c|}
\hline \multicolumn{2}{|c|}{ CÁLCULO DE ADRs A EMITIR } \\
\hline valor de la empresa calculado & $1,012,118$ \\
\hline valor en libros de los activos & 693,097 \\
\hline incremento de valor & 319,021 \\
\hline ADRs a emitir $(\mathbf{6 6 \% )}$ & $\mathbf{2 1 0 , 5 5 4}$ \\
\hline
\end{tabular}

\section{Conclusiones}

5.1. Los ADRs nivel III y nivel IV bajo la norma 144 A son una buena alternativa de financiamiento para las empresas peruanas que buscan de incrementar su capital para maximizar el valor de mercado de la empresa de acuerdo con una estructura óptima de capital.

5.2. Este mecanismo no solo permite conseguir financiamiento fuera de nuestra realidad sino que permite contar con un mercado ampliado más desarrollado y líquido donde se pueden conseguir mejores cotizaciones. Además se fortalece la imagen corporativa de la empresa emisora tanto interna como externamente.

5.3. La emisión de ADRs aumenta la posibilidad de incrementar capital de riesgo para realizar proyectos de inversión que demandan gran cantidad de recursos frescos. Es un mecanismo de fomento a la inversión directa por que podrán participar tanto inversionistas institucionales como medianos y pequeños.

\section{Recomendaciones}

6.1. Las empresas que cumplan con los requisitos exigidos por el mercado norteamericano deben emitir ADRs para lograr financiamiento en condiciones favorables para sus proyectos y mejorar su imagen internacional.

6.2. Utilizar la emisión de ADRs del NIVEL III y IV para lograr incremento de capital trayendo capital fresco de riesgo del exterior al país como inversión directa extranjera. 


\section{Pensamiento Crítico N. ${ }^{\circ}$}

\section{Bibliografía}

ALIAGA L. Rafael y Lorenzo, Sousa de Barbieri "Banca de inversión". Edit. Publicaciones Universidad de Piura, 1986.

BELLIDO S. Pedro. "Administración Financiera". Edit. Técnico Científica, 1989. Capítulo 17: Págs 245 - 272. Capítulo 18: Págs 277 - 297.

BOLTEN, Esteven. "Manual de administración financiera". Edit. Ciencia y técnica. Limusa 1987. Capítulo 8: Pags. 351 - 378. Capítulo 10: Pags. 423 - 457.

CONASEV. "Prospecto de emisión del Banco Wiese", 1994.

DIÉZ DE CASTRO, Luís y Juan, Mascarreñas. "Ingeniería financiera: la gestión en los mercados financieros internacionales”. Edit. McGRAW-HILL, 1998. Págs 91-92.

FERNÁNDEZ, Pablo. "Valoración de empresas". Edit. Gestión 2000 S.A. 1999. Capítulos 1, 14, $15,16,19$ y 20.

GLOBAL FINANCE MAGAZINE. "JP Morgan Marks 75 th Anniversary of the ADR”, 2002. Pág. 15.

GRUPO DE INVESTIGACIÓN. ALVARADO E, DELGADILLO W, GONZALEZ P, SUSANO J, UMASI S, YATACO L. Asesor: PACHECO R. ADRs "Nueva fuente de financiamiento para las empresas peruanas" 1996. Curso investigaciones económicas II. Facultad de Ciencias Económicas UNMSM.

VAN HORNE, James. "Administración Financiera". Edit. Contabilidad Moderna. Buenos Aires, 1973. Capítulo VII: Págs. 260 - 286. Capítulo VIII: Págs. 300 - 313.

http://www.pla.net.py/cnv/orientando. Glosario

http://www.superval.gob.sv/html. Glosario

http://www.corfinsura.com/español. Glosario 